Revue internationale P.M.E.

Économie et gestion de la petite et moyenne entreprise

Revue

internationale

PME

\title{
Les stratégies d'alliances des TPE artisanales
}

\section{Annabelle Jaouen}

Volume 19, numéro 3-4, 2006

URI : https://id.erudit.org/iderudit/1008503ar

DOI : https://doi.org/10.7202/1008503ar

Aller au sommaire du numéro

\section{Éditeur(s)}

Presses de l’Université du Québec

\section{ISSN}

0776-5436 (imprimé)

1918-9699 (numérique)

Découvrir la revue

Citer cet article

Jaouen, A. (2006). Les stratégies d'alliances des TPE artisanales. Revue internationale P.M.E., 19(3-4), 111-136. https://doi.org/10.7202/1008503ar

\section{Résumé de l'article}

Si les alliances stratégiques ont fait l'objet de nombreuses recherches en management stratégique, la connaissance de ce phénomène en contexte de très petite entreprise (TPE) artisanale est relativement vierge. L'objet de cet article est de montrer que l'alliance revêt des dimensions spécifiques dès lors qu'elle met en relation des toutes petites organisations. À partir de l'étude de 14 entreprises artisanales exerçant dans de multiples secteurs d'activité et d'une analyse de discours thématique, l'auteure relève deux configurations d'alliances en fonction de l'objectif du partenariat (alliance " métier " versus « produit ») et met en évidence certaines particularités : l'absence de formalisation, le refus du contrat et la prépondérance de la logique d'entraide des partenaires. Ainsi, après avoir explicité les motivations qui peuvent conduire les TPE artisanales à s'allier, il s’agit de présenter les configurations d'alliances et d'en analyser les modes de coordination. Finalement, l'auteur s'interroge sur l'apport que peut représenter le recours à l'alliance stratégique pour le développement des toutes petites structures artisanales.
Ce document est protégé par la loi sur le droit d'auteur. L'utilisation des services d’Érudit (y compris la reproduction) est assujettie à sa politique d'utilisation que vous pouvez consulter en ligne.

https://apropos.erudit.org/fr/usagers/politique-dutilisation/ 


\title{
Les stratégies d'alliances des TPE artisanales
}

Annabelle JAOUEN

Centre d'études et de recherches sur les organisations et le management (CEROM)

Groupe supérieur de commerce de Montpellier

MOTS CLÉS

\section{Alliance stratégique - Très petite entreprise artisanale - Coordination - Entraide}

\begin{abstract}
L'AUTEURE
ANNABELLE JAOUEN est professeure au Groupe supérieur de commerce de Montpellier et membre du CEROM (Centre d'études et de recherches sur les organisations et le management). Docteure en sciences de gestion de l'Université de Montpellier I, elle enseigne l'entrepreneuriat et ses travaux de recherche portent principalement sur les stratégies des très petites entreprises, ainsi que les relations de coopération dans ce contexte. Adresse: Groupe supérieur de commerce de Montpellier, 2300 avenue des Moulins, 34185 Montpellier Cédex 4. Courriel: <ajaouen@supco-montpellier.fr>.
\end{abstract}

\section{RÉSUMÉ}

Si les alliances stratégiques ont fait l'objet de nombreuses recherches en management stratégique, la connaissance de ce phénomène en contexte de très petite entreprise (TPE) artisanale est relativement vierge. L'objet de cet article est de montrer que l'alliance revêt des dimensions spécifiques dès lors qu'elle met en relation des toutes petites organisations. À partir de l'étude de 14 entreprises artisanales exerçant dans de multiples secteurs d'activité et d'une analyse de discours thématique, l'auteure relève deux configurations d'alliances en fonction de l'objectif du partenariat (alliance «métier » versus «produit») et met en évidence certaines particularités: l'absence de formalisation, le refus du contrat et la prépondérance de la logique d'entraide des partenaires. Ainsi, après avoir explicité les motivations qui peuvent conduire les TPE artisanales à s'allier, il s'agit de présenter les configurations d'alliances et d'en analyser les modes de coordination. Finalement, l'auteur s'interroge sur l'apport que peut représenter le recours à l'alliance stratégique pour le développement des toutes petites structures artisanales. 


\begin{abstract}
If strategic alliances have been largely studied by researches in strategic management, the knowledge of this phenomenon in the context of very small enterprise (VSE), especially craft industry, is relatively virgin. The purpose of this article is to show that strategic alliances present specific dimensions when they concern VSEs. Based on the study of 14 craft firms in several sectors, and a discourse analysis, results show the existence of two alliance configurations, according to partnership objective ("activity" alliance versus "product" alliance). Results also put into light several specificities of VSEs alliances: formalisation absence, contract refusal, and predominance of a mutual aid logic between the partners. Thus, after having explicated the VSEs motivation to ally, the author presents the two alliance configurations and analyses their coordination modes. Finally, the author questions the effects of strategic alliance on craft VSEs development.
\end{abstract}

\title{
RESUMEN
}

Si las allianzas estrategicas son muy estudiadas en las investigaciones en estrategia, el conocimiento de este fenómeno en el contexto de pequenitas empresas artesanales es relativamente virgen. El objeto de este articulo es de mostrar las especificidades de las allianzas estrategicas entre pequenitas empresas. Esta investigación se basa en la estudia de 14 empresas artesanales, y en una analisis de discursos. La autora identifica dos configuraciones de allianzas, segun el objetivo de la cooperación (allianza "actividad" versus allianza "producto"), y explicita algunas especificidades: la ausencia de formalización, el rechazo del contrato, y la lógica de ayuda mutua de los aliados. Asi, la autora presenta primeramente las motivaciones de las pequenitas empresas artesanales para recurrir a una allianza, y identifica despueslas configuraciones de allianzas y sus modos de coordinación. Finalmente, examina los aportes de la allianza para el desarrollo de las pequenitas empresas artesanales.

\section{ZUSAMMENFASSUNG}

Die strategischen Allianzen wurden in zahlreichen Studien des strategischen Managements untersucht. Die Kenntnisse zu Allianzen bei Mikrounternehmen (TPE, très petite entreprise) sind allerdings mangelhaft. Der vorliegende Artikel soll aufzeigen, dass strategische Allianzen eine besondere Ausprägung annehmen, sobald sie zwischen Mikrounternehmen geschlossen werden. Auf der Basis einer profunden Literaturanalyse und der Studie von 14 Mikrounternehmen aus verschiedensten Bereichen, konnten zwei spezifische Konfigurationen von strategischen Allianzen mit unterschiedlichen Zielen definiert werden: die Handwerksallianz und die Produktallianz. Dabei wurden folgende Besonderheiten festgestellt : das Fehlen jeglicher Formalisierung, Ablehnung eines Vertrages und eine selbstverständliche Hilfestellung dem Partner gegenüber. Nach der Darstellung der möglichen Gründe für Mikrounternehmen sich zusammenzuschliessen, werden die verschiedenen Konfigurationen von Allianzen dargestellt und deren Koordinationsformen analysiert. Abschliessend wird dargestellt, inwiefern sich Allianzen zur Entwicklung der Strukturen bei Mikrounternehmen eignen.

Revue internationale P.M.E., vol. 19, $\mathrm{n}^{\text {os }}$ 3-4, 2006 


\section{Introduction}

Diversifier et élargir ses débouchés, accélérer la mise sur le marché des produits, partager des informations avec d'autres entreprises, augmenter son savoir-faire et ses connaissances : autant de raisons pour lesquelles l'entreprise échange avec son environnement et, de plus en plus, avec des firmes de taille similaire par le biais d'alliances stratégiques. Les PME sont tout aussi concernées que les grandes entreprises, les secteurs industriels autant que ceux des services. Or, à la différence des grandes organisations, les PME souffrent d'une insuffisance structurelle de moyens, qui les contraint de façon permanente à concevoir leur développement sous la forme d'une alternative (Puthod, 1995). De surcroît, cette carence de ressources s'accentue avec la diminution de la taille des entreprises, les très petites entreprises (10 salariés ou moins), particulièrement les TPE artisanales, étant alors bien souvent monoproduit ou monoservice et spécialisées autour du domaine de compétences du dirigeant (Torrès, 1999).

De fait, ne pouvant pas toujours assurer leur développement de façon isolée, le recours à une alliance stratégique peut rendre possible ce développement, tout en respectant le besoin d'autonomie cher au dirigeant. En effet, l'abondante littérature sur les alliances souligne qu'elles permettent, dans une certaine mesure, le maintien de l'autonomie et qu'une inadaptation ou une insuffisance de ressources en est souvent à l'origine (Ulrich, 1983). Ce mode de développement semble donc particulièrement approprié à la toute petite organisation. Pourtant, la communauté scientifique s'est encore peu intéressée aux réponses que peut apporter l'alliance stratégique aux problèmes particuliers des très petites entreprises (TPE), car celles-ci ont jusqu'ici été rarement étudiées de façon spécifique.

Cette recherche vise donc à répondre à la problématique suivante: Quelles sont les caractéristiques, les spécificités et les apports des alliances stratégiques pour les très petites entreprises artisanales?

Par alliance stratégique, on entend un accord de coopération symétrique entre deux firmes, basé sur un contrat, formel ou non, dans le but de réaliser ensemble un projet ou d'établir une collaboration durable. Cet accord repose sur l'échange, le partage, l'acquisition ou la cession de ressources ou compétences, de façon réciproque entre les partenaires. Afin de différencier l'alliance de la coopération, il est nécessaire d'établir des critères de distinction. Ainsi, en contexte de TPE, l'alliance prend un caractère stratégique à partir du moment où (Jaouen, 2004): 
- les ressources mises en jeu ont un caractère stratégique pour l'une ou l'autre des entreprises, c'est-à-dire qu'elles sont essentielles à la survie ou au développement de la firme (p. ex., partage de brevet);

- l'objectif de la collaboration présente un caractère stratégique pour la firme;

- elle implique des changements importants dans l'organisation (structure, GRH, répartition des fonctions, etc.) ou l'activité de la firme (métier, nouveau produit, création d'un SAV);

- elle modifie le positionnement de l'entreprise ou sa représentation dans son environnement (image, mission, légitimité).

Ainsi, les alliances étudiées sont des alliances dyadiques, en fonctionnement, mettant en relation deux entreprises artisanales de moins de 10 salariés. Cette recherche vise à montrer que les alliances, lorsqu'elles concernent les TPE artisanales, relèvent de processus spécifiques, tant dans leur forme que dans leurs modes de coordination. Les résultats indiquent que les dimensions mises en évidence dans les travaux portant sur les alliances de grandes entreprises ou de PME sont dans ce cas relativement inadaptées et qu'un cadre d'analyse propre aux TPE artisanales est nécessaire.

Dans une première partie, la revue de littérature conduit à mettre en lumière les principales caractéristiques stratégiques et organisationnelles des TPE artisanales, ainsi que les enjeux que représente le recours à l'alliance dans ce contexte. La deuxième partie décrit la méthode de recherche, qualitative et basée sur l'analyse de sept cas d'alliances stratégiques, tandis que la troisième présente les résultats et leur analyse. Enfin, cet article pose la question de la spécificité des alliances en contexte de très petite entreprise.

\section{Cadre théorique}

\subsection{La TPE : un objet de recherche mal connu}

Si les très petites organisations constituent plus de $90 \%$ du tissu économique français, elles ont généralement été intégrées aux recherches portant sur les PME, en «ignorant une quelconque catégorisation pour s'intéresser aux PME en général» (Pacitto, Julien et Meier, 2002, p 35). Cependant, Torrès (2003) souligne le «grossissement» des effets avec la diminution de la taille des entreprises. En d'autres termes, plus la taille de l'entreprise est restreinte, plus les attributs généralement admis pour les PME sont accentués. Ainsi, les recherches ont admis jusqu'ici que les caractéristiques énoncées ci-après seront d'autant plus marquées que l'entreprise est de petite taille. 
La première caractéristique de la très petite entreprise tient à la simplicité de sa structure. Les TPE artisanales ne font pas exception. Cette simplicité se traduit par la faiblesse, voire l'absence de ligne hiérarchique, l'ensemble des membres étant le plus souvent directement rattaché au dirigeant. $\mathrm{Ce}$ faisant, la formalisation est rarement utilisée, la polyvalence est indispensable et les modes de communication orale et informelle sont largement privilégiés (Julien, 1990; Guéguen, 2001). De plus, les recherches sur les PME et l'information révèlent qu'elles font face à des contraintes d'accès et d'utilisation de l'information (Chapus, Lesca et Raymond, 1999), non seulement d'ordre technique (coût d'accès, difficulté à trouver l'information), mais aussi et surtout d'ordre cognitif. La prise de décision est donc largement liée à la capacité du dirigeant à traiter et exploiter l'information disponible. C'est ainsi que Chapus, Lesca et Raymond (1999), mais aussi Baillette (2000) montrent que les petites organisations tendent à développer des réseaux personnels et informels d'informations avec d'autres PME. Elles peuvent ainsi percevoir les signaux envoyés par l'environnement et être davantage au courant de ses évolutions.

Figure 1

Contraintes organisationnelles et stratégiques des PME

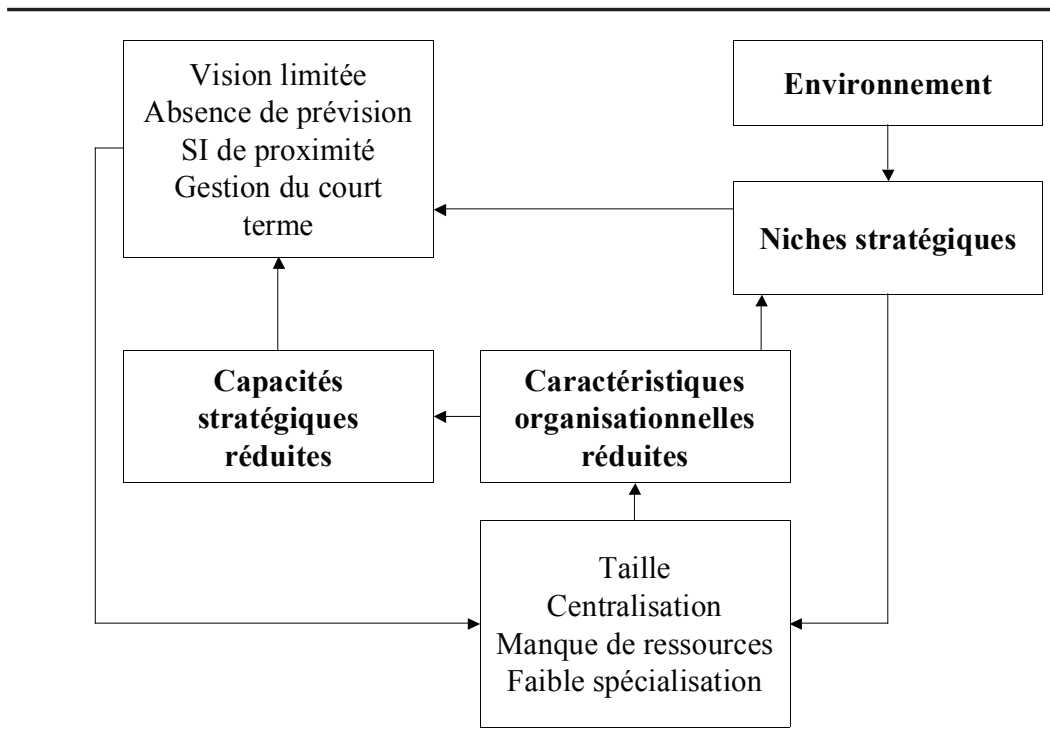

Source: Guéguen, 2001, p. 97.

Revue internationale P.M.E., vol. 19, nºs 3-4, 2006 
Deuxièmement, l'entreprise artisanale est caractérisée par un manque structurel de ressources (Chappoz, 1991; Guilhon, 1994; Imbs, 1995; Puthod, 1995 ; Asquin, Polge et Reynaud, 1999; Sauner-Leroy, 2002; McAdam, 2002). Sa petitesse implique des moyens par essence limités en quantité : ressources humaines, financières, physiques, temps, etc. Ainsi, s'il est admis que les petites entreprises artisanales manquent de moyens, elles développent en contrepartie une spécialité, concentrant ainsi leurs efforts sur un métier/produit / domaine de compétences privilégié. C'est ainsi que Polge (2003) avance que la mono-activité des petites structures est synonyme d'un seul axe stratégique pour lequel sont déployées des ressources d'ampleur limitée.

Troisièmement, la TPE artisanale est marquée par l'omniprésence de son dirigeant. Le dirigeant est certainement l'élément qui a fait l'objet du plus grand nombre de recherches sur la PME: extrême centralisation des décisions, court-termisme de la vision, aspect informel de la stratégie, (con)fusion de l'histoire de l'entreprise à l'histoire personnelle du dirigeant, etc. (Laufer, 1975 ; Carland et al., 1984 ; Carrière, 1990; Julien, 1990; Lefebvre, 1991; Marchesnay, 1992; Marmuse, 1992; Cossette, 1996; Gamot et Vidaillet, 1998; Lauriol,1998; Marchesnay, 1998; Fonrouge, 2002; Torrès, 2003 ; Jaouen, 2005, notamment). Le développement de l'entreprise étant étroitement lié aux perceptions et représentations du dirigeant, plus le dirigeant perçoit son environnement comme illisible, plus les dimensions qualitatives et la part des représentations cognitives prennent de l'importance dans la qualification de la position de son entreprise (Silvestre et Goujet, 1996). Les auteurs ont d'ailleurs relevé que, dans de nombreux cas, seuls les acteurs de proximité sont correctement repérés, une partie notoire des autres intervenants étant ignorée ou inconnue du dirigeant. Il faut alors prendre en compte la représentation que se fait le dirigeant de la réalité qui l'entoure. La représentation se définit comme un phénomène de reconstruction de la réalité, à partir de la perception de signaux extérieurs et sur la base du cadre de références et du passé de chaque individu (Silvestre et Goujet, 1996). Tyler et Steensma (1995) ont trouvé également que les décideurs n'utilisent qu'un nombre limité d'informations pour prendre les décisions parmi l'ensemble des informations disponibles : ils utilisent celles qui entrent dans leur cadre de référence et se basent sur leurs expériences passées pour évaluer les choix à poser, ce qui induit des limites cognitives importantes qui influencent la prise de décision.

Par conséquent, la TPE artisanale se caractérise par des relations fortement personnalisées à son environnement. Le premier cercle de l'environnement est constitué de toutes les parties prenantes ayant un intérêt à la bonne marche de l'entreprise: employés, propriétaires, apporteurs de capitaux, clients, fournisseurs, etc. Ce cercle constitue «l'environnement spécifié» 
de la PME (Fourcade et Marchesnay, 1997) et se traduit concrètement par des relations personnelles d'autant plus fortes que l'entreprise est petite. Il convient alors d'entretenir ces relations, où la dimension affective et intersubjective est fortement présente. Le second cercle est celui de l'environnement institutionnel: collectivités locales, administrations, syndicats professionnels, clubs de dirigeants, associations sportives et culturelles, etc. Bien souvent, le dirigeant de TPE tisse un réseau de relations institutionnelles vitales pour son affaire, car il contribue à conforter son image et sa légitimité. Il se constitue ainsi un carnet d'adresses qui, par l'information recueillie, lui permet de se tenir au courant de tous les événements susceptibles d'influencer ses propres affaires (arrivée d'un concurrent, prochain appel d'offres, etc.; Fourcade et Marchesnay, 1997). C'est ainsi que la gestion de la petite entreprise est généralement caractérisée de "gestion de proximité»: proximité géographique et relationnelle des partenaires, proximité hiérarchique, spatiale, mais aussi temporelle (Torrès, 2003), cette proximité étant d'autant plus marquée que la taille de l'entreprise est réduite.

L'ensemble de ces éléments a des conséquences importantes en matière d'alliance stratégique. En effet, la prépondérance de l'informel et de la proximité influe fortement sur la gestion de l'alliance et c'est généralement le manque structurel de ressources qui conduit les dirigeants à recourir à une alliance stratégique (Jaouen, 2005). Les travaux sur les alliances stratégiques distinguent généralement les alliances additives et les alliances complémentaires (Hennart, 1988; Dussauge et Garrette, 1995), auxquelles Puthod (1995) ajoute les alliances à caractère oblique, c'est-à-dire réalisées avec des entreprises potentiellement concurrentes sur une partie de l'activité de l'entreprise. Ce dernier montre, en outre, que les PME favorisent les alliances avec des partenaires aux ressources complémentaires et ont tendance à refuser de s'allier avec des concurrents directs. De plus, les chercheurs travaillant sur les alliances s'accordent à dire que c'est essentiellement une question de ressources qui est à l'origine du recours à l'alliance (Ulrich, 1983) : inadaptation à un besoin, insuffisance, rareté, conduisent alors les entreprises à rechercher des ressources à l'extérieur de l'entreprise.

\subsection{Les alliances stratégiques : éléments conceptuels}

Dussauge et Garrette (1995) définissent les alliances stratégiques comme «des associations entre plusieurs entreprises indépendantes qui choisissent de mener à bien un projet ou une activité spécifique, en coordonnant leurs compétences, moyens et ressources nécessaires, plutôt que $a$ ) de mettre en œuvre ce projet ou activité de manière autonome, en supportant seules les risques et en affrontant seules la concurrence, ou $b$ ) de fusionner entre elles ou de procéder à des cessions ou acquisitions d'activités » (p. 17). Pour 
Ingham (1991), les alliances stratégiques sont définies comme des «accords explicites établis dans une perspective de longue durée, par lesquels les entreprises échangent, partagent ou combinent des moyens (humains, techniques ou financiers) pour atteindre un ou plusieurs objectifs (rentabilité à long terme, croissance équilibrée, réduction des risques) afin de bâtir ou maintenir des avantages et positions concurrentiels durables» (p. 45). Ce qui distingue cette définition de celle de Dussauge et Garrette est à la fois une plus grande diversité d'objectifs et la perspective temporelle de la relation. Si ces définitions sont appropriées aux grandes entreprises, Puthod (1995) précise qu'en ce qui concerne les petites, l'alliance est un moyen de partager des ressources rendues nécessaires pour le développement de la PME dans des conditions privilégiées. L'alliance est donc une forme de rapport contractuel qui implique un certain degré d'interdépendance et qui doit durer dans le temps, en engendrant un gain pour les deux partenaires. Il considère que l'alliance stratégique repose sur le partage de ressources considérées comme essentielles: technologie, brevets, marques, outils de production, réseaux de distribution, etc. Ainsi, la firme met en jeu ce qu'elle a de plus précieux, de plus essentiel à sa survie.

Hennart (1988) classe les motifs de la coopération en deux catégories: les coopérations «d'échelle» et les coopérations de «complémentarité». Les premières ont pour objet principal la recherche d'une part de marché et d'un plus grand pouvoir; les secondes sont plus orientées vers l'accès à de nouveaux marchés ou l'utilisation de ressources et/ou de compétences que l'entreprise ne possède pas. Puthod démontre en 1995 que la majorité des PME favorisent la complémentarité. La volonté de réaliser des économies liées à la dimension ou la recherche de la taille critique semblent secondaires, exception faite des entreprises sous-traitantes contraintes par les donneurs d'ordres de se regrouper en vue d'atteindre un chiffre d'affaires minimal (hors de notre champ d'étude). Il propose ensuite (en 1996) une typologie d'alliances en contexte de PME. Il axe sa réflexion sur la valeur stratégique des ressources et sur le degré de dépendance à l'égard du partenaire. La valeur des ressources apportées par la PME conditionne le rôle plus ou moins moteur qu'on peut lui attribuer dans le développement de l'alliance: c'est ce que Puthod appelle le degré de motricité. Si ce degré est élevé, c'est que les ressources et les compétences mises en jeu sont stratégiques, c'est-à-dire qu'elles permettent aux partenaires de disposer d'un avantage concurrentiel durable. Cette motricité est cependant à relativiser en fonction du degré de dépendance. L'analyse de l'accord selon ces deux dimensions facilite l'éva- 
luation de la PME dans l'alliance. Pour ce faire, Puthod suggère une méthode relevant d'un processus de filtrage. Chaque indicateur préconisé dans le schéma présenté ci-bas joue un rôle de filtre de plus en plus contraignant.

La dépendance s'évalue en tenant compte de la configuration de l'alliance (forme et taille des partenaires) et du niveau du flux d'affaires avec le partenaire. La motricité va s'analyser relativement à deux pôles: les débouchés offerts et la nature des ressources apportées. Les débouchés potentiels sont évalués soit en termes de part de marché, soit en fonction du nombre d'applications (pour les nouvelles technologies notamment). À partir de là, l'auteur définit quatre positions possibles de la PME dans sa relation d'alliance.

\section{Figure 2}

\section{Les dimensions de l'évaluation de la position de la PME dans l'alliance}

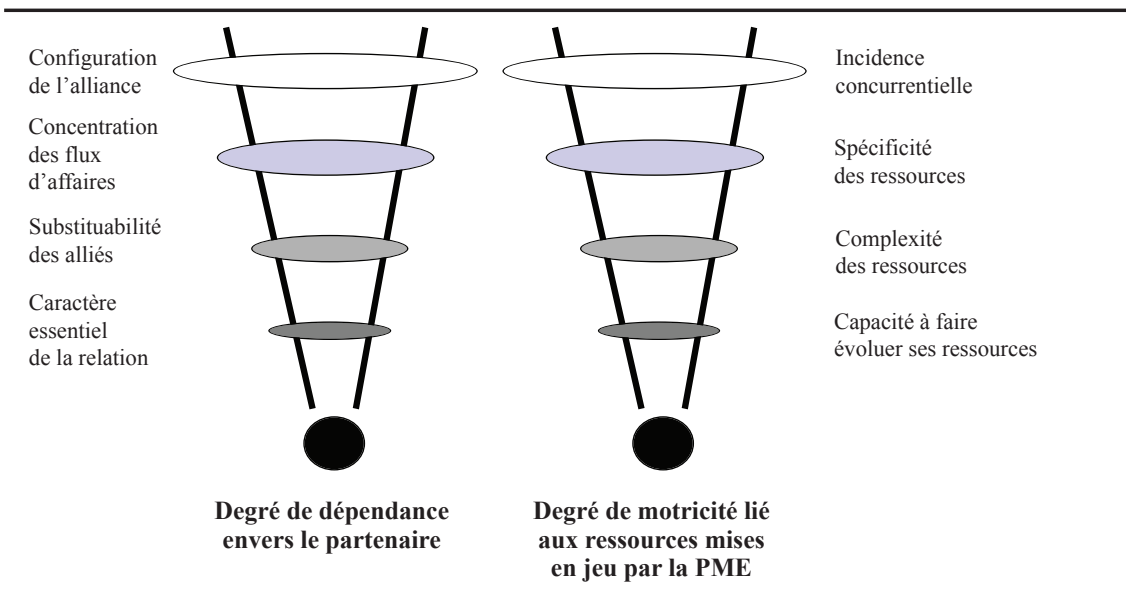

Source: Puthod, 1996, p. 3.

Revue internationale P.M.E., vol. 19, $\mathrm{n}^{\text {os }}$ 3-4, 2006 
Figure 3

Typologie d'alliances de PME

Degré de motricité

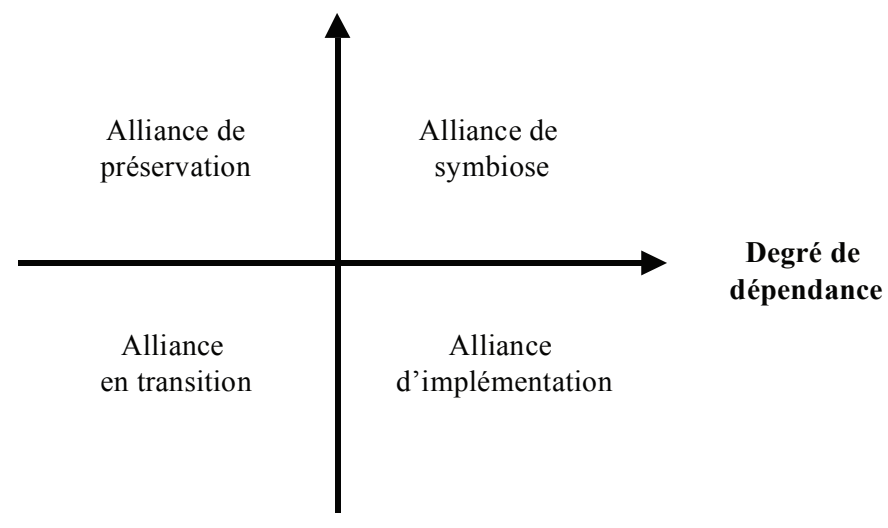

Source: Puthod, 1996, p. 7.

Les alliances en transition sont des alliances en démarrage ou en déclin, portées pour la plupart sur la commercialisation. Elles ne font pas (ou peu) l'objet de prises de participation et concernent en général plus de deux partenaires.

Les alliances d'implémentation sont basées sur une confiance très forte dès l'origine, avec un effet sur l'image très positif. Les accords sont généralement orientés vers la production et il apparaît une dissymétrie de taille entre les partenaires (GE/PMI ou MI/PI), où la PMI est en situation de dépendance forte et dont l'objet principal est de renforcer le savoir-faire. Dans ce cas, les prises de participation peuvent être importantes et ces alliances sont dyadiques.

Les alliances de symbiose concernent des entreprises de taille équilibrée et les fonctions concernées sont diverses. Deux partenaires ou plus peuvent y participer et le but est avant tout un élargissement de la gamme, un développement international et/ou un renforcement du savoir-faire.

Les alliances de préservation sont généralement des alliances centrées sur la technologie et faisant l'objet d'une confiance prudente à l'origine. Deux partenaires sont concernés, sans prise de participation. Il peut apparaître dans cette configuration des tentatives de captation du savoir-faire. 
Généralement, l'alliance stratégique est présentée dans la littérature comme une solution alternative à la croissance interne et externe (Dussauge et Garrette, 1995). Si la croissance n'est pas toujours perçue comme un objectif en soi pour les dirigeants de TPE (Marchesnay, 1982), le développement est parfois souhaité (meilleure rentabilité, augmentation des marges). Toutefois, ce développement peut être freiné par le manque de ressources de l'entreprise, celui-ci pouvant rendre la croissance impossible. C'est alors une question de ressources qui conduit les TPE à entrer dans une alliance (Jaouen, 2005). En d'autres termes, dans le cas où le dirigeant souhaite développer l'activité, élargir le métier, toucher de nouveaux clients, etc., l'entreprise ne dispose pas des ressources et / ou compétences pour le faire isolément. Cette notion est qualifiée de «saturation de ressource» (p.212). En effet, il apparaît que, bien souvent, une ressource au moins est saturée, cela signifiant que la ressource en question constitue le goulet d'étranglement de l'activité, celle dans laquelle il est nécessaire d'investir pour permettre le développement de la firme. Pour se développer, les TPE se verraient obligées d'engager des investissements dont elles n'ont pas les moyens (recrutement, achat de machine, développement du catalogue produits, etc.). Dans ce contexte, le recours à l'alliance est donc un choix profondément stratégique. En effet, ne pouvant assurer leur développement par croissance interne, les TPE vont trouver dans l'alliance une façon d'exploiter des ressources qu'elles n'ont pas les moyens d'acquérir: ressources financières, compétences techniques complémentaires, compétences commerciales, temps (Jaouen, 2004). Ainsi, si les motifs du recours à l'alliance pour les TPE sont généralement liés à une contrainte de ressources, la connaissance des spécificités des TPE artisanales dans ce contexte demeure relativement restreinte.

D’un point de vue général, la littérature met notamment en évidence a) l'influence des représentations du dirigeant sur la prise de décision, $b$ ) le caractère subjectif et intuitif de la stratégie, $c$ ) la prépondérance de la proximité en interne comme en externe, $d$ ) la faible formalisation des tâches et des activités et $e$ ) le manque structurel de ressources de la TPE. Qu'en est-il dans un contexte d'alliance? Ces caractéristiques influent-elles sur le fonctionnement des alliances stratégiques? Sur leur mode de coordination? C'est à ces hypothèses de recherche qu'il s'agit d'apporter des éléments de réponse. 


\section{Méthodologie}

Cette recherche se fonde sur 14 entretiens semi-directifs avec des dirigeants de TPE, soit l'étude de sept cas d'alliances stratégiques. L'échantillon, établi par choix raisonné (Royer et Zarlowski, 1999), comprend des entreprises exerçant dans de multiples secteurs de l'artisanat:menuiserie, facture d'orgues, pose de parquets, etc. Les entretiens ont été menés entre février et juin 2004, avec des entreprises installées en Languedoc-Roussillon. Les données collectées sont en très grande majorité des discours, discours des deux dirigeants alliés, car peu d'écrits retracent la collaboration. Nous avons parfois pu avoir accès à un certain nombre de données secondaires sur les entreprises elles-mêmes : sites Internet, plaquettes, catalogues. Le mode de collecte retenu est l'entretien individuel semi-directif, l'objectif étant de recueillir des données discursives reflétant notamment l'univers mental conscient ou inconscient des dirigeants interrogés. Pour ce faire, nous avons utilisé un guide d'entretien préstructuré, découpé en thèmes prédéfinis: l'entreprise, l'alliance, les compétences et ressources possédées et manquantes. Les entretiens ont été enregistrés sur magnétophone, afin de rendre les données collectées exhaustives et donc plus fiables, permettant des analyses de contenu plus fines. Enfin, pour éviter des conclusions biaisées par une source unique d'informations, nous avons recouru à la «double source» au sens de Baumard et al. (1999, p. 246), qui consiste à «recouper une information fournie par une source auprès d'une seconde source». Il s'agissait ici d'interviewer les deux dirigeants alliés pour «confronter» ou tout au moins comparer leurs propos.

La méthode d'analyse des données retenue est une analyse de discours, basée sur une analyse de contenu thématique (Miles et Huberman, 2003), permettant la classification des données en classes, en idéaux-types. La base de données brutes a ensuite été analysée grâce à un codage thématique et la réalisation de matrices intrasites (analyse de chaque alliance individuellement) puis intersites (analyse comparative des alliances pour relever des régularités et établir des groupes). Les matrices intrasites se composent de deux matrices centrées respectivement sur chaque partenaire puis d'une troisième matrice centrée sur l'alliance, dans laquelle sont reprises certaines informations issues des deux précédentes et où les propos des dirigeants alliés sont mis en parallèle afin d'avoir une vision globale du partenariat ${ }^{1}$. À côté de chaque thème relevé dans le discours est noté le nombre d'occurrences rencontrées, c'est-à-dire le nombre de mots ou d'unités de sens qui se rattachent à ce thème, pour avoir un aperçu de l'importance donnée à

1. L'intégralité des matrices intrasites est disponible auprès de l'auteur. La liste exhaustive des matrices n'est pas fournie en annexe pour des raisons d'anonymat des entreprises.

Revue internationale P.M.E., vol. 19, $\mathrm{n}^{\text {os }}$ 3-4, 2006 
chaque thème par le dirigeant. Parmi les 48 thèmes ayant fait l'objet d'un codage se trouvent ceux-ci: clientèle, connaissance de la concurrence, compétences possédées, compétences manquantes, ressources jugées clés, ressources saturées ou manquantes, compétences et ressources recherchées chez le partenaire, motifs de l'alliance, fonctionnement, répartition des tâches, mode de coordination, relation des dirigeants avant l'alliance, contribution perçue sur le positionnement de l'entreprise, sur le développement de ressources et compétences, modifications de la stratégie, etc.

L'objet de la recherche qualitative étant principalement la découverte, l'exploration d'un nouveau domaine et le développement d'hypothèses, elle implique donc un certain nombre de biais dont il faut tenir compte. Si l'«interprétativisme» permet une compréhension en profondeur d'un phénomène, il y a inévitablement interprétation des actions, non seulement de la part du chercheur, mais aussi des acteurs étudiés. Ainsi, un entretien sera un acte caractérisé par l'interprétation de l'interviewé puis celle du chercheur. Toutefois, la triangulation des données et l'hétérogénéité de l'échantillon permettent de penser que ces résultats présentent une validité interne et externe satisfaisante. En effet, on considère généralement qu'un échantillon homogène favorise la mise en évidence de relations et la construction d'une théorie, en évitant tout élément atypique. Par opposition, on va favoriser des éléments dissemblables lorsqu'on souhaite étendre des résultats déjà établis et possédant une forte validité interne. Entre les deux, Cook et Campbell (1979, cités par Royer et Zarlowski, 1999) proposent une solution qui consiste à utiliser des échantillons composés d'éléments volontairement très différents, pour augmenter la validité externe des résultats. En effet, «le principe d'inférence est le suivant: l'hétérogénéité exerçant une influence négative sur la significativité de l'effet, si la relation apparaît significative malgré cet inconvénient, alors les résultats peuvent faire l'objet d'une généralisation » (p. 197). Ainsi, la petitesse de l'échantillon est, dans une certaine mesure, compensée par une variété de secteurs et de profils d'entreprises. 


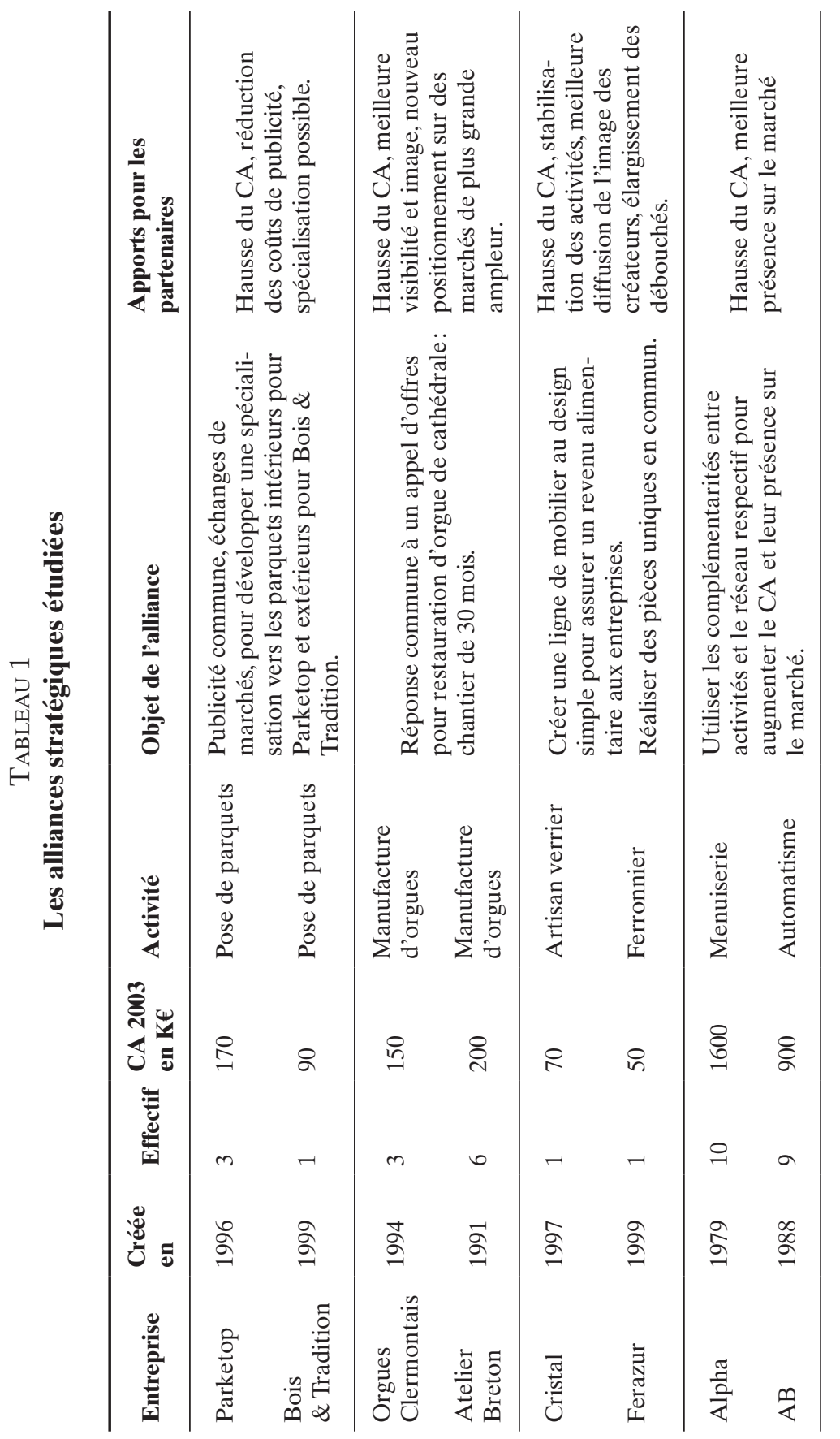

Revue internationale P.M.E., vol. 19, $\mathrm{n}^{\text {os }}$ 3-4, 2006 


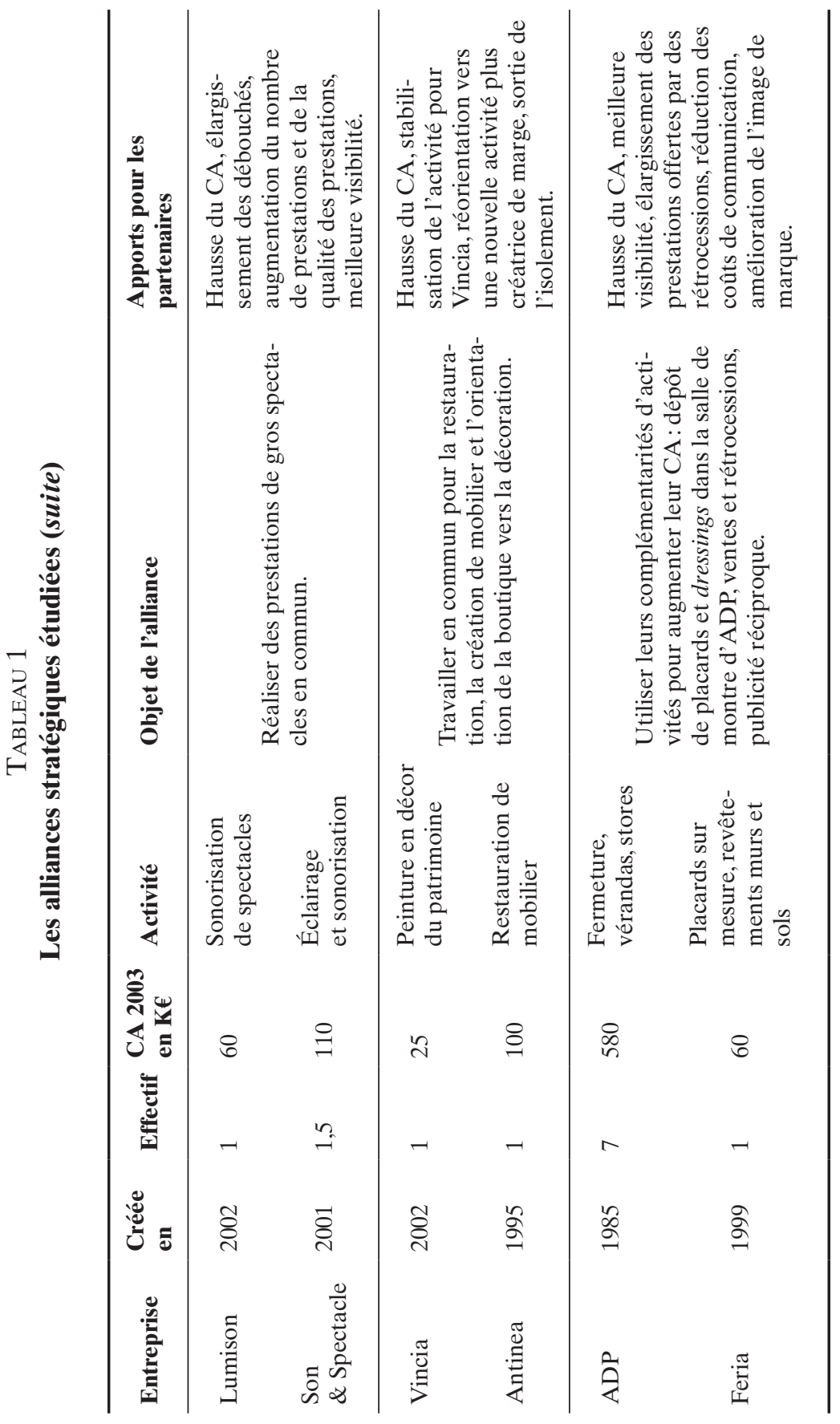

Revue internationale P.M.E., vol. 19, $\mathrm{n}^{\text {os }} 3-4,2006$ 


\section{Configuration des alliances de TPE artisanales}

L'analyse des données a permis de mettre en évidence deux grandes dimensions concernant l'objet des alliances. D'un côté, un groupe se démarque par sa volonté de développer une offre ou une activité nouvelle, par sa remise en question du métier de départ, voire par sa volonté de réorientation (qualifiée d'alliance «métier»). De l'autre, le second groupe se caractérise par un cumul de ressources complémentaires, peu d'échanges et l'atteinte d'un «effet taille» : élargir la gamme de produits ou services sans augmenter la taille des firmes (qualifiée d'alliance «produit»).

\subsection{Les alliances orientées «métier»}

L'alliance «métier» résulte de la mise en commun et/ou du partage de ressources entre les entreprises. Si les firmes conservent leur activité initiale, elles travaillent ensemble à développer une nouveauté, que ce soit une prestation commune, une enseigne, un produit ou des connaissances, afin de répondre ensemble à des besoins qu'elles ne pourraient pas satisfaire seules. Trois alliances sont concernées: Vincia/Antinea, Parketop/Bois \& Tradition et Cristal/Ferazur. Issu d'une combinaison de ressources complémentaires, ce type d'alliance résulte d'une réflexion sur le métier ou l'activité d'origine de l'entreprise. Ainsi, certaines firmes envisagent un recentrage (Parketop/Bois \& Traditrion) alors que d'autres recherchent la diversification (Vincia/Antinea et Cristal/Ferazur), mais l'ensemble des entreprises réalisant ce type de partenariat voient dans l'alliance un moyen de se réorienter.

Parketop (pose de parquets) souhaite se spécialiser dans la pose de parquets intérieurs haut de gamme et la rénovation d'escaliers pour laquelle il est dépositaire exclusif d'un brevet sur le département. Pourtant, il ne parvient pas à se spécialiser, car il n'a pas suffisamment de chantiers de ce type, ni de ressources financières, pour refuser les chantiers de parquets extérieurs (tours de piscines, terrasses). Il est contraint, par la faiblesse de ses ressources financières, de réaliser tous les chantiers qui se présentent à lui. S'il avait une trésorerie suffisante, il pourrait refuser ces contrats et consacrer du temps aux activités commerciales de prospection et de démarchage de clients pour les parquets intérieurs. Or, sa ressource financière étant «saturée», il ne peut se permettre de choisir ses chantiers. C'est au même problème que Bois \& Tradition est confrontée. Il aime particulièrement travailler à l'extérieur et, dans son idéal, il souhaiterait ne faire que des parquets extérieurs. Pourtant, pour les mêmes raisons que Parketop, il est contraint d'accepter des chantiers de parquets intérieurs. Ainsi, l'alliance consiste à s'échanger les chantiers dans

Revue internationale P.M.E., vol. 19, n ${ }^{\text {os }}$ 3-4, 2006 
le domaine de prédilection de chacun, dans le but de se spécialiser et de ne faire que les chantiers qu'ils affectionnent. Outre cette dimension d'échange, ils communiquent ensemble pour toucher, à eux deux, plus de clients.

Antinea est une entreprise artisanale de restauration de meubles. Son dirigeant, travaillant seul, a toujours eu pour passion la création de mobilier. Mais son activité n'étant pas assez rentable, il a été contraint depuis sa création à accepter des chantiers pour lesquels il n'était «pas rémunéré à sa juste valeur». C'est par hasard qu'il rencontre la dirigeante de Vincia, peintre en décor du patrimoine, venue lui amener un meuble à restaurer. En manque de travail, sa trop grande spécialisation l'empêchait de trouver suffisamment de chantiers. L'alliance consiste ici à partager la boutique d'Antinea en présentant leurs activités respectives, à développer les prestations communes et à développer une troisième activité de décoration d'intérieur. Permettant à Vincia de stabiliser son activité et de se réorienter sur une activité plus lucrative (la vente), elle permet également à Antinea, en améliorant la rentabilité et en dégageant du temps, de développer la création de meubles, ce à quoi le dirigeant aspirait.

Le cas Cristal/Ferazur diffère légèrement des précédents en ce que la nouvelle offre, résultant de la mise en commun des compétences des partenaires, n'est pas fondamentalement différente des deux activités d'origine des entreprises alliées. En effet, Cristal, artisan verrier, s'est allié avec Ferazur, ferronnier, pour développer une ligne de mobilier en commun. La particularité de ces deux entrepreneurs tient à leur caractère artistique. Tous deux ont un style très marqué et se positionnent dans le très haut de gamme et le mobilier (et objet de décoration) design. Ne faisant presque que de la pièce unique signée, leur activité ne leur apporte pas assez de clients et le prix de leurs œuvres est trop élevé pour leur assurer une présence en boutique. Ainsi, Ferazur, dans une volonté de survie et de stabilisation de son activité, est allé chercher Cristal afin de concevoir une ligne de mobilier simple et compétitive, de développer la présence en boutique (sur la Côte d'Azur et Paris essentiellement), mais aussi de créer des pièces uniques alliant les deux matières: fer et verre. Dans ce cas d'alliance, si la recherche d'une activité plus lucrative et plus rentable est évidente, la diversification est quant à elle moins frappante, étant donné que l'offre ne bouleverse pas fondamentalement les activités d'origine. On peut néanmoins considérer qu'il s'agit d'une diversification dans le sens où les dirigeants passent d'une conception «improvisée » et «artistique» de pièce unique, à une rationalisation et une production en petite série de meubles, pour une cible totalement distincte de leur cible d'origine. 
Cette première catégorie d'alliances est alors marquée par la volonté de repenser son métier et de créer une offre ou une activité nouvelle. Elle fonctionne par la mise en commun, le partage ou l'échange de ressources et de compétences entre les dirigeants, qui gardent pour la plupart une activité propre et différente de cette nouvelle offre commune. C'est une démarche volontaire, de co-construction, qui lie les deux partenaires. En effet, ils repensent leur métier ensemble, voire envisagent une réorientation complète de l'activité en commun. Ainsi, l'idée étant de devenir plus efficaces ou plus rentables, les entreprises sont parfois prêtes à investir dans des ressources communes: recrutement ou achat commun de matériel. Cette catégorie s'oppose à une autre forme d'alliance, l'alliance orientée «produit », qui relève de motivations relativement opposées à l'alliance «métier».

\subsection{Les alliances orientées «produit»}

L'alliance orientée «produit» peut s'illustrer par les propos d'un des dirigeants interrogés:

Être tout petit, c'est bien parce qu'on est très spécialisé et très compétent sur un domaine, mais sur un domaine seulement. Et les clients, en général, ils ont un besoin qui dépasse un peu votre seul domaine de compétences. Donc, vous vous retrouvez face à un dilemme: perdre le client parce que vous ne savez pas faire, ou récupérer le client et vous débrouiller pour trouver la bonne personne capable de répondre à ce besoin. Et dans ce deuxième cas de figure, comme en général vous n'avez pas les moyens d'embaucher quelqu'un, trouver un partenaire, une entreprise qui a les bonnes compétences, celles qui complètent les vôtres, c'est la solution idéale. Vous avez l'efficacité, la réactivité, sans les coûts.

Par ces propos, ce dirigeant résume parfaitement la situation dans laquelle se trouvent les entreprises qui réalisent des alliances «produit». Comme son nom l'indique, ce type d'alliance est orienté vers le produit, le marché ou, plus largement, vers l'image véhiculée par l'entreprise dans son environnement. En effet, il n'y a pas de réelle remise en cause de l'activité de départ, mais plutôt un souhait de paraître plus grand aux yeux du client, d'être compétitif face à des concurrents plus gros, en d'autres termes, d'atteindre un «effet taille», de «croître sans grandir». L'alliance consiste ici en une addition de ressources complémentaires et non plus en une combinaison de ces ressources. C'est ainsi que les entreprises vont développer une enseigne commune, un catalogue commun, ou encore répondre à des appels d'offres ensemble, puis simplement se répartir le travail et réaliser chacune la partie pour laquelle elle est spécialisée. Quatre alliances sur sept entrent dans cette catégorie: ADP/Feria, Orgues Clermontais/Atelier Breton, Alpha/AB, et Lumison/Son \& Spectacle. 
L'alliance Orgues Clermontais / Atelier Breton est assez illustrative de cette catégorie. En effet, ces deux manufactures d'orgues ont pour activité la restauration et la fabrication d'orgues de cathédrales et d'églises. Leur alliance consiste en la réponse commune à un appel d'offres de restauration d'un très grand orgue de cathédrale, nécessitant 30 mois de travail. Ce chantier aurait été, pour les deux entreprises, irréalisable. À titre d'illustration, le CA dégagé par ce chantier représente plus de trois fois le CA annuel d'Orgues Clermontais. De plus, un tel chantier nécessite un «plateau technique » qu'une entreprise seule ne peut posséder, en termes d'assise financière, mais aussi de matériel et de main-d'œuvre. Tous les salariés de cette entreprise sont mobilisés pour sa réalisation. Dans ce cas d'alliance, le métier n'est pas remis en question et les compétences nécessaires à la réalisation du projet sont déjà possédées par les deux firmes. C'est une question de «taille critique» (ici entendue comme la taille suffisante pour répondre à cet appel d'offres) qui est au centre de l'alliance. Par la somme de leurs deux structures et de leurs ressources (financières, matérielles et humaines), les entreprises ont la taille nécessaire pour réaliser ce chantier. Le dirigeant d'Orgues Clermontais l'exprime bien: «Techniquement, [tous seuls] on ne peut pas répondre sur ce marché, parce qu'on n'a pas la structure et qu'on ne peut pas assurer les délais. Et donc, ma foi, on a deux solutions. Soit on ne répond pas, soit on crée un partenariat. Et à ce moment-là, le cumul de nos CA et du plateau technique [...] nous permet de répondre dans de bonnes conditions.» Ainsi, une fois l'appel d'offres gagné, les entreprises se répartissent le travail et chacune rentre dans ses ateliers pour restaurer sa partie: tuyauterie et acoustique pour Atelier Breton, mécanique et menuiserie pour Orgues Clermontais. Mais les dirigeants admettent qu'ils auraient très bien pu inverser les parties à restaurer. Ce cas illustre bien l'idée que pour «paraître » plus gros et pour accéder à certains chantiers, l'alliance est nécessaire et implique une addition de ressources, sans forcément partage ou mise en commun.

D’une façon générale, ces alliances visent à élargir les prestations offertes au client afin de répondre à des besoins plus diversifiés. L'alliance a donc pour effet majeur de véhiculer une image d'entreprise plus grande que ce qu'elle n'est en réalité, sans pour autant utiliser les bénéfices de l'alliance pour investir dans la croissance interne de l'entreprise. Il semble alors que la firme qui a recours à une alliance de ce type veut s'ouvrir de nouvelles opportunités de marché sans pour autant augmenter sa taille. Par un système de répartition des tâches et de rétrocession financière, les entreprises peuvent conserver leur spécialité tout en externalisant vers le partenaire la partie moins maîtrisée et en touchant des commissions sur les ventes au prorata de leur participation. Ainsi, si l'alliance métier s'oriente vers une réorganisation 
de l'entreprise ou une réflexion sur le métier, l'alliance produit vise davantage à améliorer la présence commerciale ou à trouver de nouveaux débouchés pour les compétences actuelles de l'entreprise.

\section{Caractéristiques des alliances entre TPE artisanales}

\subsection{Des alliances informelles}

Lorsqu'ont été abordées les questions concernant la coordination des partenaires, plusieurs points sont apparus. Tout d'abord, aucune alliance n'est formalisée par un contrat écrit. L'engagement est (m)oral entre les dirigeants, qui mettent en avant l'importance de la parole donnée et des valeurs communes. Mais si ce résultat constitue déjà en soi une particularité des TPE, cela va plus loin dans le sens où le contrat est catégoriquement refusé par 12 dirigeants sur 14 . Trois arguments au refus ont été majoritairement cités: la volonté de conserver son autonomie, l'importance de la parole donnée et l'importance des valeurs communes. Si le contrat était proposé, il pourrait engendrer une rupture de la confiance mutuelle. La représentation que se fait le dirigeant du contrat pourrait devenir autoréalisatrice et le pousser ainsi à adopter un comportement opportuniste... D'une façon générale, la contractualisation, généralement présentée avec la confiance comme garantes d'une alliance pérenne, prend une dimension toute différente en contexte de TPE artisanale, puisque la contractualisation peut être perçue à l'inverse de ce qu'elle est en grande entreprise: une forme de trahison risquant de mettre en péril la pérennité du partenariat ou, en tout cas, de déstabiliser la relation (Jaouen, 2004). Voici ce que le dirigeant d'Alpha, la menuiserie en alliance avec $\mathrm{AB}$, en pense:

Non, il ne faut pas en faire. Dès qu'il y a un contrat, il y a des contraintes, il y a des règles. Et il ne faut pas de règles. Il faut que ça reste complètement informel. Il ne faut pas lier les hommes sur des sentiments ou des valeurs. À partir du moment où il y a un contrat, $[\ldots]$ on peut facilement se retrancher derrière. Et nous, c'est plus profond que ça. Il faut que ce soit un état d'esprit. C'est pour ça qu'on ne peut pas formaliser, réglementer ce type de relation. Si vous l'amenez sous forme de contrat ou de règles, c'est fini, c'est mort, ça n'existera plus.

Ainsi, la confiance entre les dirigeants prédomine et les conduit à refuser le contrat, car la relation amicale rend la contractualisation superflue.

\subsection{Des alliances d'entraide}

Si la confiance prédomine largement et le contrat est refusé, celui-ci est jugé inutile, car l'importance de la parole et des valeurs communes ainsi que la 
volonté d'autonomie prennent le pas sur un contrat que beaucoup admettent ne pas avoir les compétences pour constituer. Les résultats de cette recherche montrent qu'à la relation économique, ou relation «d'affaires», se superpose une relation sociale, «humaine», fondée sur des liens forts (au sens de Granovetter, 1985). Dans les discours, des items tels que «ensemble», «en commun », «soutien» ou «collaboration» apparaissent fréquemment. Ainsi, si l'objet premier du partenariat est d'ajouter ou de combiner des ressources complémentaires, le but est également d'évoluer ensemble et de valoriser le partenaire. Les dirigeants expriment régulièrement le fait qu'ils se recommandent mutuellement aux clients, qu'ils se font de la publicité réciproque et qu'ils se stimulent et s'encouragent. La relation humaine tient alors une part prépondérante dans le fonctionnement de l'alliance et le co-développement est au cœur du partenariat. Le dirigeant de Orgues Clermontais exprime l'importance de travailler ensemble et en confiance: «Là, j'engage mon entreprise, tous mes salariés, la survie de ma société dans ce chantier. Il dure presque trois ans vous imaginez? Là, je n'ai pas droit à l'erreur, c'est pour ça que c'est avec lui ou personne.» Un second exemple est celui d'Antinea:

Ce qui m'a manqué jusqu'à présent, c'est ça. C'est d'avoir quelqu'un à côté avec qui l'on puisse avancer. J'avais trop de choses à faire tout seul ici,j'étais seul et [...] j'ai fait l'essentiel, à savoir bâtir, avancer... mais je n'ai pas suffisamment réfléchi à comment développer vraiment mon activité. Je n'avais pas le temps de réfléchir à ça. Je n'allais qu'à l'essentiel, [...] faire l'urgent. [...] Aujourd'hui on a vraiment une démarche un peu plus profonde du fonctionnement de l'entreprise... du développement de l'entreprise.

Mais parmi les exemples les plus frappants de cette catégorie, on trouve Alpha (menuiserie), qui a aidé $\mathrm{AB}$ (automatisme et fermeture) à se développer au démarrage et qui voit avant tout dans ce partenariat le plaisir de travailler ensemble et de le voir évoluer. C'est ainsi qu'il déclare:

Il y a eu ce petit nouveau qui s'est installé, avec qui il y a eu cette affinité et avec qui on a fait un partenariat un peu informel. Moi, avec les années, j'ai un certain relationnel et une certaine notoriété dans la région. Et comme je sentais qu'il y avait du professionnalisme, de la compétence et tout ça, je lui ai fait profiter de tout mon relationnel. Alors je lui ai ouvert un tas de portes qui sont intéressantes et où il faut souvent des années pour pouvoir accéder à ce type de marché. Pour rentrer dans certaines collectivités, pour rentrer sur certains marchés qui sont assez difficiles parce qu'ils sont déjà occupés par des entreprises [...] Il lui aurait fallu peut-être 15 ans pour arriver là où il est aujourd'hui s'il avait été tout seul [...] Il a évolué très très vite. Et sûrement. Sans accident. Parce qu'il y a cette recommandation, ce parrainage, qui est là.

Son partenaire illustre également cette notion d'entraide par les propos suivants :

Revue internationale P.M.E., vol. 19, nºs 3-4, 2006 
Demain on m'appelle, il y a une casse dans un magasin, je vais chez Alpha, je lui dis «tu peux fabriquer ça [une porte] en catastrophe?» S'il le faut je vais aller l'aider le soir après cinq heures, parce qu'il ne pourra pas me le fabriquer. Je sais qu'il me filera un coup de main pour le débiter [...] et le lendemain soir on peut poser la porte chez le client [...]. C'est un énorme plus, tout seul je ne pourrais pas faire ça. Pas aussi rapidement. Impossible.

Ainsi, les alliances stratégiques entre TPE artisanales sont marquées par une logique d'entraide très forte et la relation humaine devient tout aussi importante que le développement du chiffre d'affaires ou de la réactivité des entreprises.

\section{Conclusion}

Les résultats de cette recherche témoignent de la spécificité des alliances stratégiques lorsqu'elles mettent en relation de très petites entreprises artisanales. L'état de l'art présenté au début de ce travail a fait ressortir que les alliances stratégiques, lorsqu'elles concernent les grandes entreprises, peuvent prendre plusieurs formes: additives ou complémentaires, entre entreprises concurrentes ou non (Hennart, 1988; Dussauge et Garrette, 1995, entre autres). Les travaux sur les alliances de PME montrent en revanche que ce type de firme favorise les alliances complémentaires plutôt qu'additives (Puthod, 1995; Bacus Montfort, 1997). Les résultats de cette recherche révèlent que les stratégies d'alliances de TPE artisanales présentent la double dimension additivité et complémentarité, dimensions qui sont généralement dissociées, voire opposées dans les travaux sur les alliances entre grandes entreprises ou PME. En effet, les firmes recherchent, dans tous les cas, des ressources complémentaires aux leurs. De plus, dans les alliances «produit» notamment (mais cela est également vrai dans certaines alliances orientées «métier»), les entreprises recherchent l'atteinte d'un «effet taille» : elles cherchent à croître, sans grandir. En additionnant des ressources complémentaires, les entreprises cherchent à élargir leur gamme de prestations, à toucher un éventail plus large de clients, à agrandir leur zone de couverture et à donner l'image d'une entreprise de plus grande taille. Pouvant répondre à des demandes plus larges, dépassant leur seul domaine de compétences, elles accèdent à des marchés qu'elles n'auraient pas pu toucher si elles étaient restées seules, car leurs compétences trop spécialisées, ou leur taille trop réduite, ne leur permettent pas d'y répondre. Ainsi, les alliances stratégiques entre TPE artisanales sont spécifiques dans le sens où elles ne relèvent ni des processus mis en avant pour les grandes entreprises, ni de ceux concernant les PME. Ici, additivité et complémentarité ne s'opposent pas dans deux formes d'alliances bien distinctes, mais sont combinées afin d'atteindre le double objectif de conservation de la spécialité et d'effet taille. 
En outre, les alliances entre TPE artisanales relèvent également de modes de coordination spécifiques. Aucune alliance ne fait l'objet d'un contrat écrit et le contrat est souvent refusé. Cela est à rapprocher du lien fort et affectif établi entre les dirigeants, qui ne peuvent travailler sans confiance pour la grande majorité d'entre eux. Ainsi, dans la majeure partie des cas, la construction d'un lien fort entre les partenaires est indispensable pour envisager un partenariat et, de fait, la relation étant interpersonnelle, le contrat devient superflu.

D'un point de vue pratique, les résultats de la recherche montrent que l'alliance semble bénéfique à la TPE artisanale dans le sens où elle lui assure de nouveaux débouchés, qu'ils soient purement commerciaux ou aillent jusqu'à la remise en question de l'activité même de l'entreprise. Au vu des discours des dirigeants interrogés, l'alliance permet d'améliorer la pérennité des firmes. Oliver (2001) a établi une corrélation entre l'absence d'alliance et la mort précoce des petites entreprises de biotechnologie. Si ce secteur d'activité n'est pas concerné par la présente recherche, plusieurs dirigeants témoignent néanmoins du rôle joué par l'alliance dans leur pérennité. Par ailleurs, l'alliance permet à la TPE de dépasser la saturation d'au moins une ressource, celle qui l'empêche, par sa nature ou son volume, de se développer isolément (Jaouen, 2004, 2005). Outre les ressources financières, plusieurs dirigeants ont constaté que l'alliance leur a permis d'éviter de recruter, un tel recrutement étant trop lourd en termes de trésorerie, de coût, d'organisation, ou même n'étant tout simplement pas souhaité. L'évitement de capacité ainsi créé a permis à un certain nombre d'entreprises de se développer de façon plus souple et plus stable à la fois, le partenariat étant plus flexible que le coût fixe engendré par un recrutement.

D’un point de vue général, l'alliance stratégique, dès lors qu'elle met en relation des entreprises artisanales, semble présenter des caractéristiques particulières qui rendent inappropriés les référentiels utilisés pour analyser les alliances de grandes entreprises. Les résultats présentés dans ce travail visent donc à mettre en exergue la nécessité d'adopter un cadre d'analyse adapté aux problématiques particulières de ce type de firme, où la rationalité économique n'est pas toujours présente, mais où il est nécessaire de raisonner en termes de rationalité «élargie». 


\section{Bibliographie}

Asquin, M., M. Polge et E. Reynaud (1999), «L'entrepreneur créateur de ressources? Une contribution empirique », $\mathrm{I}^{\text {er }}$ Congrès de l'entrepreneuriat, Lille.

Bacus Montfort, I. (1997), Modélisation du choix de partenaires dans la coopération entre PMI, Thèse de doctorat, Université Paris IX.

BAILlETte, P. (2000), L'importance des activités relationnelles pour l'aide à la décision:le cas de l'adhésion du propriétaire-dirigeant de PME à une association de dirigeants, Thèse de doctorat en sciences de gestion, Université de Montpellier II.

Baumard, P., C. Donada, J. Ibert et J.-M. Xuereb (1999), «La collecte des données et la gestion de leurs sources», dans R.A. Thiétart (dir.), Méthodes de recherche en management, Paris, Dunod, p. 224-256.

Carland, J.W., F. Hoy, W.R. Boulton et J.A.C. Carland (1984), «Differentiating entrepreneurs from small business owners: a conceptualization », Academy of Management Review, vol. 9, n² 2, p. 354-359.

CARrière, J.-B. (1990), «La vision stratégique en contexte de PME: cadre théorique et étude empirique», Revue internationale PME, vol. 3, nos 3-4, p. 301-325.

Chappoz, Y. (1991), «La gestion de l'interactivité entreprise/ environnement», Revue internationale PME, vol. 4, n 3, p. 53-75.

Chapus, E., H. Lesca et L. Raymond (1999), «Collective learning within an environmental scanning coalition of small regional firms: toward a modelization», Proceedings of the 44th Annual World Conference of the International Council of Small Business, Naples, Italie.

Cossette, P. (1996), «La vision stratégique du propriétaire-dirigeant de PME: étude de cartographie cognitive», Revue internationale PME, vol. 9, n 1, p. 123-142.

Dussauge, P. et B. Garrette (1995), Les stratégies d'alliances, Paris, Éditions d'Organisation.

Fonrouge, C. (2002), «L'entrepreneur et son entreprise: une relation dialogique», Revue française de gestion, vol. 138, p. 145-158.

Fourcade, C. et M. Marchesnay (1997), Gestion de la PME/PMI, Paris, Nathan.

Gamot, G. et B.Vidaillet (1998), «Parole et pensée dans l'action du dirigeant», dans H. Laroche et J.-P. Nioche (dir.), Repenser la stratégie, Paris, Vuibert.

Granovetter, M. (1985), «Economic action and social structure: the problem of embeddedness », American Journal of Sociology, vol. 91, n 3, p. 481-510.

GuÉGuen, G. (2001), Environnement et management stratégique des PME: le cas du secteur Internet, Thèse de doctorat en sciences de gestion, Université de Montpellier I.

Guilhon, A. (1994), «Le rôle de l'investissement intellectuel dans les stratégies d'implantation", Revue internationale PME, vol. 7, $\mathrm{n}^{\text {os }}$ 3-4, p. 147-171.

Hennart, J.F. (1988), «A transaction cost theory of equity joint ventures», Strategic Management Journal, vol. 9, $\mathrm{n}^{\circ}$ 4, p. 361-374.

Revue internationale P.M.E., vol. 19, $\mathrm{n}^{\text {os }}$ 3-4, 2006 
Ingham, M. (1991), «La perception du succès des alliances stratégiques », Revue internationale $P M E$, vol. 4, nº 2, p. 43-84.

ImBs, P. (1995), «Le financement des PME innovatrices: le temps du partenariat», Revue française de gestion, vol. 104, p. 30-45.

JAOUEN, A. (2004), «Spécificités des alliances stratégiques dans le cas des très petites entreprises », Revue de l'économie méridionale, vol. 52, n 208, p. 263-281.

JAOUEN, A. (2005), Les alliances stratégiques entre très petites entreprises: un cadre d'analyse, Thèse de doctorat en sciences de gestion, Université de Montpellier I.

Julien, P.-A. (1990), «Vers une typologie multicritère des PME», Revue internationale $P M E$, vol. 3, nos 3-4, p. 411-425.

LAufer, J.-C. (1975), «Comment on devient entrepreneur», Revue française de gestion, vol. 2, p. 3-15.

LAuriol, J. (1998), «Une nouvelle approche de la décision stratégique », Revue française de gestion, vol.121, p. 65-79.

Lefebvre, E. (1991), «Profil distinctif des dirigeants de PME innovatrices», Revue internationale PME, vol. 4, $\mathrm{n}^{\circ}$ 3, p. 7-26.

Marchesnay, M. (1982), «Pour un modèle d'hypofirme», dans G. Legris (dir.), Entreprise et organisations, Paris, Economica, p. 71-90.

Marchesnay, M. (1992), «La PME: une gestion spécifique?», Problèmes économiques, vol. 2276.

Marchesnay, M. (1998), «Confiances et logiques entrepreneuriales», Économies et Sociétés, coll. «Sciences de Gestion», vol. 8-9, p. 99-117.

Marmuse, C. (1992), Politique générale: langages, intelligence, modèles et choix stratégiques, Paris, Economica.

McAdAM, R. (2002), «Large scale innovation-reengineering methodology in SME's », International Small Business Journal, vol. 20, nº 1, p. 33-52.

Miles, A.M. et A.M. Huberman (2003), Analyse des données qualitatives, Bruxelles, De Boeck Université.

Oliver, A.L. (2001), «Strategic alliances and the learning life-cycle of biotechnology firms », Organization Studies, vol. 22, n 3, p. 467-490.

Pacitto, J.-C., P.-A. Julien, et O. Meier (2002), «Les TPE sont-elles spécifiques? », Piccola Impresa/Small Business, $\mathrm{n}^{\circ}$ 2, p. 35-61.

PuthoD, D. (1995), Les alliances de PME: stratégie de développement et implications managériales, Thèse de doctorat, Université de Savoie.

PuthoD, D. (1996), «Comprendre les alliances de PME (à partir d'une base de connaissances construite sur les préoccupations de l'entrepreneur)», Ve Conférence de l'AIMS, Lille.

Polge, M. (2003), «Petite entreprise et stratégie de terroir», Revue française de gestion, vol. 29, n 144, p. 181-194.

Revue internationale P.M.E., vol. 19, nºs 3-4, 2006

(C) 2006 - Presses de l'Université du Québec

Édifice Le Delta I, 2875, boul. Laurier, bureau 450, Québec, Québec G1V 2M2 • Tél.: (418) 657-4399 - www.puq.ca 
Royer, I. et P.ZARLowski (1999), «Échantillons », dans R.-A.Thiétart (dir.), Méthodes de recherche en management, Paris, Dunod, p. 188-223.

SAUner-Leroy, J.-B. (2002), «Allocation de ressources, avantage concurrentiel et performance des PME de l'industrie manufacturière française», Revue internationale PME, vol. 15, $\mathrm{n}^{\circ}$ 1, p. 65-85.

Silvestre, H. et R. Goujet (1996), «Lisibilité de l'environnement, management stratégique: éléments de recherche sur les PMI», Revue internationale PME, vol. $9, \mathrm{n}^{\circ} 1$, p. 61-78.

Torrès, O. (1999), Les PME, Paris, Flammarion.

Torrès, O. (2003), «Petitesse des entreprises et grossissement des effets de proximité», Revue française de gestion, vol. 29, n 144, p. 119-138.

Tyler, B.B. et H.K. STEENSMA (1995), «Evaluating technological collaborative opportunities: a cognitive modeling perspective», Strategic Management Journal, numéro spécial (été), vol. 16, p. 43-70.

UlRich, D. (1983), «Governing transactions: a framework for cooperative strategy », Human Resource Management, vol. 22, nos 1-2, p. 23-40. 